\title{
Comparación de perfiles de ansiedad matemática entre estudiantes mexicanos y estudiantes alemanes
}

Cristina Eccius-Wellmann, Antonio G. Lara-Barragán, Bastian Martschink y Stefan Freitag

\section{RESUMEN}

Se determinan los perfiles de ansiedad matemática de estudiantes mexicanos y estudiantes alemanes por medio de la aplicación de un cuestionario desarrollado ex profeso. La ansiedad matemática se define en términos de tres descriptores: creencias, actitudes y emociones. Los autores definen un Índice de Ansiedad Matemática, al aplicarlo encuentran que los estudiantes alemanes presentan un valor mayor de dicho Índice, lo que significa que tienen una mayor nivel de ansiedad matemática. Las diferencias en los valores del Índice de Ansiedad Matemática se interpretan en términos de diferencias culturales. La interpretación parece ser consistente con las diferencias culturales y se ajusta a los resultados obtenidos.

Palabras clave: ansiedad matemática, diferencias culturales, estudiantes alemanes, estudiantes mexicanos.

Cristina Eccius-Wellmann

ceccius@up.edu.mx Mexicana. Doctora en Educación Matemática por la Universidad de Hamburgo, Alemania. Profesora investigadora de la Escuela de Ciencias Económicas y Empresariales, Universidad Panamericana, campus Guadalajara, México. Temas de investigación: errores algebraicos y su procedencia, enseñanzaaprendizaje de las matemáticas, ansiedad matemática y confianza en la matemática y en las TIC.

Antonio G. Lara-Barragán

alara@up.edu.mx Mexicano. Maestro en Física por la Universidad Autónoma Metropolitana, México, y maestro en Pedagogía por la Universidad Panamericana, México. Profesor investigador de la Facultad de Ingeniería, Universidad Panamericana, campus Guadalajara, México. Temas de investigación: didáctica de la física, enseñanza de las ciencias y evaluación de los aprendizajes.

\section{Bastian Martschink}

Bastian.Martschink@h-brs.de Alemán. Doctor en Electrónica. Profesor en la carrera de Ingeniería Mecatrónica, Hochschule Bonn-RheinSieg, University of AppliedScience, Sankt Augustin, Alemania. Temas de investigación: investigación en el campo de la didáctica, la teoría de probabilidad y estadística.

\section{Stefan Freitag}

Stefan.Freitag@h-brs.de Alemán. Dipl.-Berufspäd. Profesor adjunto para Asignaciones Especiales y Profesor adjunto en Matemáticas para la Escuela de Administración en la Hochschule Bonn-Rhein-Sieg University of Applied Science, Sankt Augustin, Alemania. Temas de investigación: didáctica. 


\section{Comparação de perfis de ansiedade matemática entre estudantes mexicanos e} estudantes alemães

\section{RESUMO}

Se determinam os perfis de ansiedade matemática de estudantes mexicanos e estudantes alemães por meio da aplicação de um questionário desenvolvido especificamente para isso. A ansiedade matemática se define em termos de três descritores: crenças, atitudes e emoções. Os autores definem um Índice de Ansiedade Matemática, ao aplicá-lo deparam que os est udantes alemães apresentam um valor maior de dito Índice, o que significa que têm um maior nível de ansiedade matemática. As diferenças nos valores do Índice de Ansiedade Matemática se interpretam em termos de diferenças culturais. A interpretação parece ser consistente com as diferenças culturais e se ajusta aos resultados obtidos.

Palavras chave: ansiedade matemática, diferenças culturais, estudantes alemães, estudantes mexicanos.

\section{A comparison of mathematics anxiety profiles between Mexican and German students}

\section{ABSTRACT}

This article outlines the mathematics anxiety profiles of Mexican and German students by means of a questionnaire that has been drawn up ex professo. The mathematics anxiety can be defined around three description terms: beliefs, attitudes and emotions. The authors establish a Mathematics anxiety index and when they put it into practice they observe that the German students tend to obtain a higher value, which means that they have a higher level of mathematics anxiety. The differences in the values of the Mathematics anxiety index can be understood in terms of cultural differences. This interpretation can be consistent with the cultural differences and matches the obtained results.

Key words: mathematics anxiety, cultural differences, German students Mexican students.

Recepción: 13/03/16. Aprobación: 23/02/17. 


\section{Introducción}

La ansiedad matemática (AM) ha llegado a ser un tema importante dentro del ámbito de la enseñanza de las matemáticas puesto que una gran cantidad de trabajos de investigación sugieren que tal situación representa una de las más serias dificultades para el aprendizaje y el desempeño académico satisfactorio en todos los niveles educativos. Este problema puede observarse prácticamente en todo el mundo aunque para cada región del planeta sus características varían. Sin embargo, a fin de cuentas, minimizar la AM es uno de los más grandes retos para la educación. Para lograrlo, es menester conocer sus características propias en cada región, en cada país, en cada ciudad y en cada institución educativa para así poder diseñar cursos, actividades y recursos ad hoc, con el propósito de alcanzar mejores resultados en el rendimiento académico de los estudiantes.

En particular, conocer las diferencias comparativas entre los perfiles de AM para diferentes países puede redundar en el diseño de mejores programas educativos que permitan resultados convenientes en el tema de movilidad estudiantil. De aquí surge el presente estudio por medio del cual analizamos las diferencias entre los perfiles de AM en términos de índices de AM de estudiantes mexicanos y estudiantes alemanes, considerando algunas diferencias culturales de ambos países, México y Alemania. Los estudios se realizaron en una universidad de la zona metropolitana de la ciudad de Guadalajara, Jalisco, México y en una universidad de la ciudad de Bonn, Alemania, para responder a las siguientes preguntas de investigación:

1. ¿Podría haber influencia cultural en la AM para estudiantes alemanes y estudiantes mexicanos?

2. ¿Cómo se comparan las medias de los índices de AM entre estudiantes de ambos países?

3. Para estudiantes de Administración e Ingenierías, ¿cómo se comparan las medias de los índices de AM?
Comenzaremos con una discusión sobre algunos tópicos relacionados con las características culturales que influyen en la vida cotidiana y académica de los estudiantes mexicanos y de los estudiantes alemanes; en seguida presentamos un breve estado del arte en el tema de la AM; posteriormente ponemos a consideración la manera en se obtuvieron los perfiles de AM y, finalmente, discutimos las diferencias encontradas entre tales perfiles.

\section{Fundamentos teóricos}

En muchos países se tienen planes educativos prioritarios para fomentar y promover el aprendizaje de las matemáticas (Huntsinger y col., 1997). Para ponerlos en marcha y obtener resultados satisfactorios, es necesario minimizar el gran obstáculo representado por la AM (Xie y Leong, 2008) puesto que muchos estudios argumentan que ésta y el rendimiento académico están inversamente relacionados (Caballero y col., 2008). Entendemos que la AM es una manifestación emotiva desagradable que tiene sus raíces en el miedo de entrar en contacto con las matemáticas, lo que incluye clases, tareas y exámenes (Eccius-Wellmann y Lara-Barragán, 2016).

Xie y Leong (2008), después de estudiar grupos de estudiantes caucásicos y chinos, proponen diferentes clases de ansiedad de acuerdo con características culturales diferentes. Sus categorías de ansiedad incluyen ansiedad de estado, ansiedad de cualidad y ansiedad debida a evasión social y angustia social. Sus resultados muestran que los estudiantes chinos presentan una mayor tendencia hacia la AM que los estudiantes caucásicos.

Por otro lado, otros estudios muestran que las características culturales juegan un papel importante para el aprendizaje de aspectos tales como razonamiento justificativo, comunicación y estilos de aprendizaje (Soong y col., 2012). Estos resultados los obtuvieron al comparar estudiantes de Australia y de Malasia a partir de cuestionarios diseñados ex profeso. Luego, en otro ambiente académico, Engelhard 
(2001) encontró diferencias entre estudiantes de América y de Tailandia debidas, principalmente, a la influencia materna. Engelhard también encontró que la AM y el rendimiento académico se encuentran en relación inversa y que los estudiantes americanos tienden más a la ansiedad que los estudiantes tailandeses. En otro aspecto, Huntsinger y col. (1997) compararon cuantitativamente los aprendizajes entre estudiantes chino-americanos y estudiantes taiwaneses, y entre estudiantes euroamericanos y estudiantes taiwaneses por medio de un examen elaborado para tal fin. Sus resultados muestran que los estudiantes chino-americanos y los estudiantes taiwaneses obtuvieron mejores calificaciones que los estudiantes euroamericanos. Este resultado sugiere que las diferencias culturales también influyen en el rendimiento académico, aseveración que se fundamenta en el trabajo de Hofstede, Hofstede y Minkov (2010), quienes aseguran que las diferencias culturales pueden explicar diversas cuestiones que, tanto las personas como las organizaciones, enfrentan dentro de las sociedades. El estudio de Hofstede y colaboradores se basa en cuatro dimensiones que representan otras tantas categorías universales para caracterizar sociedades: distancia de poder, evasión de incertidumbre, individualismo versus colectivismo, y masculinidad versus feminidad.

El término distancia de poder se refiere a un hecho fundamental de la brecha que puede existir en toda organización jerárquica en cuanto al poder que tiene cada estrato. Trata esencialmente sobre la forma en que una sociedad maneja las relaciones de poder establecidas a través de los valores de los superiores y sus subordinados. Esta dimensión se contempla en tres niveles: subordinados que perciben que su jefe toma sus decisiones de manera autocrática o paternalista (persuasiva); la percepción de los subordinados de que los empleados en general (sus colegas) sienten temor ante la posibilidad de discordar con sus superiores; y subordinados quienes no prefieren un jefe que tome sus decisiones de manera consultiva, sino que prefieren un jefe que decide autocrática o paternalistamente. En el ámbito académico podemos hacer la analogía de la figura del profesor como el jefe y los estudiantes como los subordinados.

El término evasión de incertidumbre puede describirse por medio de dos actitudes: sentirse nervioso o tenso en el trabajo, y la afirmación de que las reglas no deben romperse, aun cuando la persona piense que es por el mejor interés de la institución. En cuanto al ámbito académico, las actitudes mencionadas se identifican como la ansiedad ante el trabajo y el cumplimiento del reglamento impuesto tanto por las autoridades universitarias como por el profesor en el aula.

El individualismo indica la relativa importancia que en el trabajo tienen aspectos personales como el tiempo, la libertad y los retos, y la poca importancia relativa de la capacitación profesional, el uso de habilidades, de las condiciones físicas y de los beneficios. Entonces, el individualismo se centra en alcanzar metas para las que el individuo es un agente activo contra aquellas en las que el individuo depende de la institución en cuanto a recibir capacitación, a usar sus habilidades, así como que se le proporcionen condiciones de trabajo y beneficios. Las características en el ámbito académico referentes al individualismo vs. colectivismo se encuentran en la tabla 2.

La masculinidad indica la importancia relativa de aspectos del trabajo como ingresos, reconocimiento, ascensos y retos, y la poca importancia relativa de la relación con el superior, la cooperación, el área de residencia y la seguridad de empleo. Todas ellas en relación con el patrón dominante de roles de género encontrados en un gran número de sociedades, por ejemplo, que los niños se socializan hacia la autoafirmación y autoconfianza, mientras que las niñas hacia la crianza, la educación y la responsabilidad. Para el ámbito académico las características académicas referentes a masculinidad contra feminidad se resumen en la tabla 3. 
A cada una de las dimensiones anteriores se les asigna un índice numérico (tabla 5). El valor de este índice indica el grado de presencia de las características que definen a las dimensiones listadas en las tablas 1 a 4, por país (Hofstede y col., 2010).

Tabla 1. Interpretaciones para el índice de Distancia de Poder

\begin{tabular}{|l|l|}
\hline \multicolumn{1}{|c|}{ Índice pequeño para Distancia de Poder } & \multicolumn{1}{|c|}{ Índice grande para Distancia de Poder } \\
\hline 1.1 Los padres tratan a sus hijos como iguales & Los padres enseñan a sus hijos a obedecer \\
\hline 1.2 Los estudiantes tratan a sus profesores como iguales & $\begin{array}{l}\text { Los estudiantes muestran respeto a sus profesores, aun } \\
\text { fuera de clase }\end{array}$ \\
\hline $\begin{array}{l}1.3 \text { Los profesores esperan iniciativas de sus estudiantes durante } \\
\text { la clase }\end{array}$ & $\begin{array}{l}\text { Los profesores deberían tomar todas las iniciativas durante } \\
\text { la clase }\end{array}$ \\
\hline $\begin{array}{l}1.4 \text { Los profesores son expertos que transfieren verdades } \\
\text { impersonales }\end{array}$ & Los profesores son gurús que transfieren sabiduría personal \\
\hline $\begin{array}{l}1.5 \text { La calidad del aprendizaje depende de la comunicación en dos } \\
\text { sentidos y de la excelencia de los estudiantes }\end{array}$ & $\begin{array}{l}\text { La calidad del aprendizaje depende de la excelencia del } \\
\text { profesor }\end{array}$ \\
\hline $\begin{array}{l}1.6 \text { Las personas menos educadas poseen valores más autoritarios } \\
\text { que las personas educadas }\end{array}$ & $\begin{array}{l}\text { Las personas con mayor educación y las de menor } \\
\text { educación muestran igualdad de valores autoritarios }\end{array}$ \\
\hline $\begin{array}{l}1.7 \text { Las políticas educativas se dirigen principalmente a las escuelas } \\
\text { de educación media }\end{array}$ & $\begin{array}{l}\text { Las políticas educativas se dirigen principalmente a las } \\
\text { universidades }\end{array}$ \\
\hline
\end{tabular}

Fuente: Elaboración propia.

Tabla 2. Individualismo

\begin{tabular}{|l|l|}
\hline \multicolumn{1}{|c|}{ Colectivista } & \multicolumn{1}{c|}{ Individualista } \\
\hline 2.1 Los estudiantes hablan sólo cuando el grupo lo autoriza & $\begin{array}{l}\text { Se espera que los estudiantes hablen individualmente en } \\
\text { clase }\end{array}$ \\
\hline 2.2 El propósito de la educación es aprender a hacer & El propósito de la educación es aprender a aprender \\
\hline 2.3 Los títulos proporcionan acceso a grupos de mayor estatus & $\begin{array}{l}\text { Los títulos incrementan el valor económico y/o el } \\
\text { autorrespeto }\end{array}$ \\
\hline
\end{tabular}

Fuente: Elaboración propia. 
Tabla 3. Masculinidad

\begin{tabular}{|l|l|}
\hline \multicolumn{1}{|c|}{ Femenino } & \multicolumn{1}{c|}{ Masculino } \\
\hline $\begin{array}{l}\text { 3.1 El estudiante promedio es la norma; encomio a los estudiantes } \\
\text { débiles }\end{array}$ & $\begin{array}{l}\text { Los mejores estudiantes es la norma; encomio a los } \\
\text { estudiantes excelentes }\end{array}$ \\
\hline 3.2 Envidia a los estudiantes sobresalientes & Competencia en clase; se trata de sobresalir \\
\hline 3.3 Reprobar es un incidente menor & Reprobar es un desastre \\
\hline 3.4 Los estudiantes subestiman su propio desempeño: discreción & $\begin{array}{l}\text { Los estudiantes sobrestiman su propio desempeño: } \\
\text { alimentan su ego }\end{array}$ \\
\hline 3.5 Se valora la cordialidad de los profesores & Se admira la genialidad de los profesores \\
\hline 3.6 Hombres y mujeres estudian, en parte, las mismas asignaturas & Hombres y mujeres estudian diferentes asignaturas \\
\hline 3.7 Hombres y mujeres enseñan a niños pequeños & Las mujeres enseñan a los niños pequeños \\
\hline
\end{tabular}

Fuente: Elaboración propia.

\section{Tabla 4. Evasión de incertidumbre}

\begin{tabular}{|l|l|}
\hline \multicolumn{1}{|c|}{ Evasión de incertidumbre débil } & \multicolumn{1}{c|}{ Evasión de incertidumbre fuerte } \\
\hline $\begin{array}{l}\text { 4.1 Los estudiantes se sienten cómodos con situaciones de } \\
\text { aprendizaje de respuesta abierta y se interesan por buenas } \\
\text { discusiones }\end{array}$ & $\begin{array}{l}\text { Los estudiantes se sienten cómodos con aprendizajes } \\
\text { estructurados y se interesan por la respuestas correctas }\end{array}$ \\
\hline 4.2 Los profesores pueden decir "no lo sé" & Se espera que los profesores tengan todas las respuestas \\
\hline 4.3 Los resultados se atribuyen a la propia habilidad de la persona & Los resultados dependen de circunstancias y buena suerte \\
\hline 4.4 Los profesores involucran a los padres & Los profesores informan a los padres \\
\hline
\end{tabular}

Fuente: Elaboración propia.

Tabla 5. Índices numéricos para las cuatro dimensiones

\begin{tabular}{|l|c|c|c|}
\hline & Distancia de poder & $\begin{array}{c}\text { Evasión de } \\
\text { incertidumbre }\end{array}$ & Individualismo \\
\hline Alemania & 35 & 65 & 67 \\
\hline México & 81 & 82 & 30 \\
\hline
\end{tabular}

Fuente: Elaboración propia. 
Por otra parte, McSweeney (2002) aduce algunas limitaciones del trabajo de Hofstede y colaboradores, en el sentido de que sus resultados y argumentos sobre conceptos cruciales tal como el de cultura, adolecen de defectos por la metodología empleada para su obtención. Sin embargo, para el propósito de nuestro trabajo, algunas de las limitaciones mencionadas por McSweeney representan situaciones ventajosas. Tal es el caso para el que Hofstede clama sobre la cultura nacional como la dimensión compartida por todos los individuos en una nación, aunque sus análisis se refieren a sitios localizados. Por ello, más bien habría que hablar de resultados aplicables "microlocalmente".

De acuerdo con lo anterior, las dimensiones de Hofstede y colaboradores pueden ser aplicadas a los entornos universitarios en los que realizamos nuestro estudio. Las universidades se convierten así en sitios localizados de aplicabilidad, tanto de las dimensiones de Hofstede como de las componentes de ansiedad matemática que describimos en la siguiente sección. Las primeras se han seleccionado de la amplia gama de descriptores de las dimensiones determinadas por Hofstede y colaboradores de manera que, en nuestra experiencia, se ajustan a rasgos culturales compartidos por nuestros estudiantes.

Otros aspectos de la AM han sido estudiados por pedagogos, psicólogos, matemáticos y expertos que combinan estos campos (Macías-Martínez y Hernández-Pozo, 2008). Una revisión detallada de la literatura se ha realizado previamente (Eccius y Lara, 2016), por lo que mencionaremos brevemente algunos de los autores que consideramos más importantes. Legg y Locker (2009) definen la AM como un temor o tensión generalizada asociada con circunstancias que involucran interacción con las matemáticas. Mencionan, también, que la AM puede ser un factor decisivo como para que los estudiantes eviten cursos de matemáticas o carreras profesionales con un número alto de asignaturas relacionadas con matemáticas. Leppävirta (2011) asegura que la
AM es un sentimiento de tensión que interfiere con un buen número de manipulaciones algebraicas y solución de problemas. Otro punto de vista es el de Fiore (1999), quien reporta que la evidencia sugiere que la AM se debe, principalmente, a la forma en que se presentan los contenidos de las asignaturas de matemáticas y no a los contenidos mismos, conclusión que se apoya en la afirmación de Sloan (2010), quien asevera que los profesores tienen ansiedad, por lo que actúan como sus portadores y se la transfieren a sus alumnos.

Nuestra propia experiencia como profesores en el nivel superior y como tutores (o asesores) académicos por más de dos décadas se ajusta mejor a lo reportado por Hembree (1990), en el sentido de que la AM significa temor a involucrarse con actividades matemáticas tales como asistencia a clases teóricas, tareas y exámenes. Este concepto también tiene un soporte fisiológico por los estudios realizados por Macías-Martínez y Hernández-Pozo (2008) cuyos resultados muestran correlación entre las mismas manifestaciones fisiológicas asociadas con el miedo y las manifestaciones de los alumnos que enfrentan exámenes y nuevos cursos de matemáticas.

Finalmente, hemos encontrado en la literatura que los conceptos asociados con la AM se definen de manera conveniente de acuerdo con un criterio dado. Como lo menciona Crechtley (citado por Goos, Brown y Makar, 2008), la terminología se usa, con frecuencia, de manera ambigua y con diferentes significados acordes con cada caso particular. Así, con esto en mente, en la siguiente sección introducimos los conceptos que usamos en nuestro proyecto.

\section{Definición de las componentes de Ansiedad Matemática}

Definimos la AM en términos de las siguientes tres categorías: creencias, actitudes y emociones (Gil y col., 2005). Para nuestros propósitos, definimos a las creencias como el conocimiento subjetivo adquirido a través de experiencias relacionadas con las 
matemáticas mismas y con los entornos familiares y sociales. Luego, siguiendo a McLeod (1989) y a Gómez Chacón (2000), dividimos a las creencias en cuatro subcategorías: a) sobre su enseñanza y aprendizaje; b) sobre el individuo mismo como aprendiz; c) sobre el contexto social, y d) sobre la naturaleza del conocimiento y el proceso de aprendizaje.

Las actitudes conforman una categoría más compleja que las creencias por las numerosas definiciones encontradas en la literatura. Mato y de la Torre (2009) piensan que una actitud es una disposición positiva o negativa ante una situación particular, mientras que Gil y colaboradores (2005) afirman que una actitud es una predisposición evaluativa que determina las intenciones personales e influencian el comportamiento. Estas ideas concuerdan con las de Mohamed y Waheed (2011) y las de Hannula (2002) en el sentido de que las actitudes son evaluaciones que guían el comportamiento de una persona, que se desarrollan y cambian al transcurrir el tiempo. Estos conceptos tienen en común que las actitudes se refieren a comportamientos observables dicotómicos relacionados con aceptación o rechazo, respeto o irreverencia, y curiosidad o indiferencia. De acuerdo con esto, utilizaremos el término actitud como comportamiento o expresión verbal en consonancia con una creencia o una emoción adquirida durante el proceso de aprendizaje y moldeada por los entornos familiar, académico y social (Guerrero y col., 2001). Como consecuencia solamente consideraremos dos formas de actitud: positiva y negativa.

Las emociones se conciben como respuestas a estímulos internos y externos que tienen una influencia directa sobre el individuo que las experimenta. Nos parece evidente que las respuestas a estímulos provocan dos clases de cambios: fisiológicos y cognoscitivos (Damasio, 2003); esto es, los estímulos se experimentan tanto física como mentalmente. De acuerdo con Hannula (2002), las emociones no son observables, a menos de que tengan un alto grado de intensidad conjuntando tres factores independientes: respuesta fisiológica (como, por ejemplo, una descarga de adrenalina), expresiones faciales (sonreír o fruncir el ceño) y experiencias subjetivas (tristeza o alegría). También se observa que muchos estudiantes son conscientes de sus emociones, por lo que son capaces de expresarlas libremente o de controlarlas.

La descripción anterior de los descriptores creencias, actitudes y emociones permite pensar que ninguna de ellas está aislada, sino que están interrelacionadas.

\section{Metodología}

El propósito de nuestro estudio es encontrar (o no encontrar) diferencias significativas entre los Índices de Ansiedad Matemática obtenidos para los estudiantes alemanes (A) y los estudiantes mexicanos (M), y dar explicaciones posibles de tales diferencias. También se pretende analizar cuáles de los factores, emociones, actitudes y/o creencias, son los que pudieran aportar o no a una diferencia en el Índice de Ansiedad Matemática de los estudiantes encuestados de ambos países.

El cuestionario original, de 30 preguntas, fue una composición obtenida de dos cuestionarios diferentes, el primero debido a Galbraith y Haines (2000) y el segundo desarrollado por Pierce, Stacey y Barkatsas (2007). Un análisis estadístico por medio de la alfa de Cronbach y de un análisis factorial redujo el cuestionario a 20 preguntas, con los siguientes tres factores: Actitudes, x1, x4, x13, x16, x17, x18, x19 y x20; Creencias: x3, x6, x7, x9, x11, x12 y x15, y Emociones: $\mathrm{x} 2, \mathrm{x} 5, \mathrm{x} 7, \mathrm{x} 9, \mathrm{x} 10$ y x14 (Eccius y Lara, 2016). Los ítems $\mathrm{x} 7 \mathrm{y} \mathrm{x} 9$ se repiten en las creencias y las emociones, ya que en el análisis factorial quedaron con cargas factoriales fuertes en ambos factores. Para el estudio por factores, se tomarán en cuenta ambos.

El cuestionario elaborado y validado por Eccius y Lara (2016) se aplicó a 419 estudiantes de una universidad situada en la zona metropolitana de Guadalajara, Jalisco, México, y a 247 estudiantes alemanes 
de una universidad situada en Bonn, Alemania. En ambos casos, el número de estudiantes es el total de alumnos inscritos en dos áreas, Administración e Ingeniería en los primeros dos semestres de sus respectivas carreras. En lo que sigue utilizaremos la siguiente notación: AB y MB indican estudiantes alemanes de administración y estudiantes mexicanos de administración respectivamente, mientras que AE y ME indican estudiantes alemanes de ingeniería y estudiantes mexicanos de ingeniería respectivamente. La distribución se muestra en la tabla 6 .

El cuestionario tiene una escala de Likert para medir actitudes, emociones y creencias. La escala es: casi nunca (1), a veces (2), la mitad de las veces (3), con frecuencia (4), casi siempre (5). Para la alfa de Cronbach y el Índice de Ansiedad Matemática, los ítems negativos (x2, x6, x9, x10, x11, x12 y x17) se calcularon nuevamente en una escala de Likert inversa; esto es, si un estudiante respondía 1, el nuevo cálculo producía un 5. Los coeficientes de la alfa de Cronbach se calcularon (SPSS, versión 22) para medir la consistencia interna y confiabilidad de cada uno de los grupos, A y M, AB y MB, AE y ME.

\section{El Índice de Ansiedad Matemática}

Los valores del índice de AM se encuentran en un intervalo de 0 a 1 . El cero representa la ausencia de ansiedad, mientras que el 1 representa la máxima presencia de ansiedad. Hemos calculado un índice de AM para cada estudiante alemán denotados por el subíndice i, y un índice para cada estudiante mexicano, denotado por el subíndice j. Como se mencionó con anterioridad, los ítems negativos se recalcularon en una escala inversa de Likert, por lo que todos los ítems contribuyen, de manera positiva, a un Índice de NO Ansiedad Matemática, en los cálculos. La suma de todas las respuestas a todos los ítems de cada estudiante se calculó con la notación SUMi y SUMj. Así, debido a que la suma mínima que puede tener un estudiante dado es 20 (20 ítems en escala de Likert del 1 al 5) y la suma máxima es 100, el Índice de Ansiedad Matemática, por estudiante, se encuentra con las ecuaciones:

Para estudiantes alemanes:

$$
I_{i}=1-\frac{S U M_{i}}{80}
$$

Para estudiantes mexicanos:

$$
I_{j}=1-\frac{S U M_{j}}{80}
$$

Cada grupo de estudiantes tiene un valor promedio del índice de AM, el cual se obtiene de la suma de todos los índices Ii de los estudiantes del grupo en cuestión, dividido entre el número de miembros de tal grupo. Por ejemplo, el índice (AT) de AM para estudiantes alemanes es:

$$
A T=\frac{\sum_{i=1}^{i=24} I_{i}}{247}
$$

Por consiguiente, tenemos seis promedios diferentes de índices de AM: AT, MT, ABI, MBI, GEI y MEI, los índices promedio para estudiantes alemanes y mexicanos, para estudiantes alemanes de

Tabla 6. Distribución de estudiantes alemanes y mexicanos cursando las carreras de Administración e Ingeniería

\begin{tabular}{|l|c|c|c|}
\hline & $\begin{array}{c}\text { Número total } \\
\text { de estudiantes } \\
\text { encuestados }\end{array}$ & $\begin{array}{c}\text { Número de } \\
\text { estudiantes de } \\
\text { administración }\end{array}$ & $\begin{array}{c}\text { Número de } \\
\text { estudiantes de } \\
\text { ingeniería }\end{array}$ \\
\hline Alemania & $247(\mathrm{~A})$ & $122(\mathrm{AB})$ & $125(\mathrm{AE})$ \\
\hline México & $419(\mathrm{M})$ & $289(\mathrm{MB})$ & $130(\mathrm{ME})$ \\
\hline
\end{tabular}

Fuente: Elaboración propia. 
administración, para estudiantes mexicanos de administración, para estudiantes alemanes de ingeniería y para estudiantes mexicanos de ingeniería, respectivamente.

De la misma manera se encuentran los índices por factor, es decir, por creencias, actitudes y emociones. Estos índices contribuyen al índice de AM global.

\section{Hipótesis}

Como hipótesis nula (Ho) se establece que el promedio del índice de AM para los estudiantes alemanes es menor o igual al promedio de estudiantes mexicanos en los tres distintos grupos, por ejemplo: $\mathrm{AT} \leq \mathrm{MT}$. Se realizó una prueba de t-Student para detectar si las diferencias eran estadísticamente distintas. La prueba es a una cola y la hipótesis nula se rechaza con un nivel de confianza del 95\%, aceptando la hipótesis alterna (Ha): AT>MT. Se realizó el mismo estudio para cada uno de los pares, según su rama de estudio, AB vs. MB y AE vs. ME.

También se realizó para los promedios de los índices de contribución a la AM, debido a los distintos factores; creencias, actitudes y emociones, la prueba de diferencias de medias. Para GAT vs. GMT, AAT vs. AMT, EAT vs. EMT; donde la primera letra corresponde al factor: $\mathrm{C}$ de creencias, A de actitudes y E de emociones, mientras las siguientes dos letras siguen teniendo el mismo significado. Así también se realizó un estudio de comparación de medias de los grupos por rama de estudio: $\mathrm{CAB} v s$. CMB, AAB $v s$. AMB, EAB vs. EMB, CAE vs. CME, AAE vs. AME y finalmente, EAE vs. EME.

\section{Resultados}

Los resultados del análisis de alfa de Gronbach se resumen en la tabla 7.

Los valores de la tabla 7 indican una buena confiabilidad del test.

La tabla 8 muestra los promedios de los índices de AM de todos los grupos sin desglosar por factor y la comparación de medias, así como rechazos y aceptaciones de las correspondientes hipótesis.

Para todos los grupos de comparación, los niveles de significancia indican el rechazo de la hipótesis nula y la aceptación de la hipótesis alterna, es decir, los promedios de los índices de AM de los estudiantes de México son significativamente menores a los promedios de los índices de AM de los estudiantes de Alemania.

Tabla 7. Análisis de la alfa de Cronbach

\begin{tabular}{|c|c|}
\hline Grupo de estudiantes & Alfa de Cronbach \\
\hline A & 0.944 \\
\hline AE & 0.917 \\
\hline AB & 0.956 \\
\hline M & 0.932 \\
\hline$M E$ & 0.918 \\
\hline$M B$ & 0.911 \\
\hline
\end{tabular}

Fuente: Elaboración propia. 
Se calcularon las medias de los índices por factor. Cada índice aportará al índice de AM, ya que la AM se compone de los tres factores: creencias, actitudes y emociones.

La tabla 9 muestra las medias de los índices por factor del total de los estudiantes alemanes y mexicanos, AT y MT respectivamente.

Para las tres pruebas t de igualdad de medias, las varianzas se asumen iguales. Dada la significancia de la prueba t de Student, se rechaza la hipótesis nula de igualdad de medias. En ambas comparaciones $\mathrm{CAT}>\mathrm{GMT}$, AAT>AMT.

Las medias de los índices de las emociones, son estadísticamente iguales, EAT=EMT.

La tabla 10 muestra las medias de los índices por factor de estudiantes de administración alemanes y mexicanos, $\mathrm{AB}$ y $\mathrm{MB}$ respectivamente.
En todos los índices por factor, no hay igualdad de varianza, lo cual fue considerado en la prueba $t$ de comparación de medias. Tanto la media de los índices de creencias como de las actitudes fueron distintos estadísticamente entre los estudiantes alemanes de administración y los estudiantes mexicanos de administración, $\mathrm{CAB}>\mathrm{CMB}$ y $\mathrm{AAB}>\mathrm{AMB}$. La media del índice de las emociones no mostró diferencia estadísticamente significativa.

En la tabla 11 se muestran las medias de los índices por factor de estudiantes de ingenierías alemanes y mexicanos, AE y ME respectivamente.

Para las tres pruebas, las varianzas se asumen iguales, dados los valores de la prueba de Levene. Las medias de los índices comparados de estudiantes de ingeniería alemanes y mexicanos, AE y ME, respectivamente, son estadísticamente distintos. $\mathrm{CAE}>\mathrm{CME}$; AAE $>$ AME y EAE $>$ EME.

Tabla 8. Estudio estadístico de las medias de los índices de AM de los diferentes grupos de comparación

\begin{tabular}{|c|c|c|}
\hline & Indices de Ansiedad Matemática & p valor \\
\hline Estudiantes alemanes (AI) & 0.4445 & $\begin{array}{c}\text { Se rechaza } \mathrm{H}_{0} \text {, que } \\
\mathrm{Al} \leq \mathrm{Ml}\end{array}$ \\
\hline Estudiantes mexicanos (MI) & 0.3726 & $\begin{array}{c}\text { y se acepta } \mathrm{H}_{\mathrm{a}} \\
\text { Al > MI }\end{array}$ \\
\hline $\begin{array}{l}\text { Estudiantes alemanes de } \\
\text { Administración (ABI) }\end{array}$ & 0.4884 & $\begin{array}{c}\text { Se rechaza } \mathrm{H}_{0} \text {, que } \\
\qquad A B I \leq \mathrm{MBI}\end{array}$ \\
\hline $\begin{array}{l}\text { Estudiantes mexicanos de } \\
\text { Administración (MBI) }\end{array}$ & 0.4080 & $\begin{array}{c}\text { y se acepta } \mathrm{H}_{a} \\
\qquad A B I>M B I\end{array}$ \\
\hline $\begin{array}{l}\text { Estudiantes alemanes de } \\
\text { Ingeniería (AEI) }\end{array}$ & 0.4017 & $\begin{array}{c}\text { Se rechaza } \mathrm{H}_{0} \text {, que } \\
\mathrm{AEI} \leq \mathrm{MEI}\end{array}$ \\
\hline $\begin{array}{l}\text { Estudiantes mexicanos de } \\
\text { ingeniería (MEI) }\end{array}$ & 0.2938 & $\begin{array}{c}\text { y se acepta } \mathrm{H}_{\mathrm{a}} \\
\text { AEI > MEI }\end{array}$ \\
\hline
\end{tabular}

Fuente: Elaboración propia. 
Tabla 9. Resultados de la comparación de las medias de los índices por factor del total de los estudiantes

\begin{tabular}{|c|c|c|c|c|c|c|}
\hline & Grupo & $N$ & $\begin{array}{c}\text { Media de los } \\
\text { índices por factor }\end{array}$ & $\begin{array}{l}\text { Desviación } \\
\text { estándar }\end{array}$ & $\begin{array}{l}\text { Prueba de Levene } \\
\text { Significancia }\end{array}$ & $\begin{array}{l}\text { Prueba t para } \\
\text { igualdad de medias } \\
\text { (significancia bil.) }\end{array}$ \\
\hline \multirow{2}{*}{ Creencias } & СAT & 247 & 0.4787 & 0.2627 & \multirow[t]{2}{*}{0.204} & \multirow[t]{2}{*}{0.000} \\
\hline & CMT & 419 & 0.4016 & 0.2488 & & \\
\hline \multirow{2}{*}{ Actitudes } & AAT & 247 & 0.4679 & 0.2013 & \multirow[t]{2}{*}{0.347} & \multirow[t]{2}{*}{0.000} \\
\hline & AMT & 419 & 0.3705 & 0.1917 & & \\
\hline \multirow{2}{*}{ Emociones } & EAT & 247 & 0.4679 & 0.2337 & \multirow[t]{2}{*}{0.071} & \multirow[t]{2}{*}{0.111} \\
\hline & EMT & 419 & 0.3705 & 0.2227 & & \\
\hline
\end{tabular}

Fuente: Elaboración propia en SPSS.

Tabla 10. Resultados de la comparación de las medias de los índices por factor de los estudiantes de Administración

\begin{tabular}{|c|c|c|c|c|c|c|}
\hline & Grupo & $N$ & $\begin{array}{l}\text { Media de los } \\
\text { índices por factor }\end{array}$ & $\begin{array}{l}\text { Desviación } \\
\text { estándar }\end{array}$ & $\begin{array}{l}\text { Prueba de Levene } \\
\text { Significancia }\end{array}$ & $\begin{array}{l}\text { Prueba t para } \\
\text { igualdad de medias } \\
\text { (significancia bil.) }\end{array}$ \\
\hline \multirow{2}{*}{ Creencias } & CAB & 122 & 0.5383 & 0.2921 & \multirow[t]{2}{*}{0.003} & \multirow[t]{2}{*}{0.001} \\
\hline & CMB & 289 & 0.4397 & 0.2499 & & \\
\hline \multirow{2}{*}{ Actitudes } & $A A B$ & 122 & 0.4985 & 0.2298 & \multirow[t]{2}{*}{0.001} & \multirow[t]{2}{*}{0.000} \\
\hline & AMB & 289 & 0.4057 & 0.1935 & & \\
\hline \multirow{2}{*}{ Emociones } & EAB & 122 & 0.4204 & 0.2599 & \multirow[t]{2}{*}{0.002} & \multirow[t]{2}{*}{0.064} \\
\hline & EMB & 289 & 0.3701 & 0.2255 & & \\
\hline
\end{tabular}

Fuente: Elaboración propia en SPSS.

Tabla 11. Resultados de la comparación de las medias de los índices por factor de los estudiantes de Ingeniería

\begin{tabular}{|c|c|c|c|c|c|c|}
\hline & Grupo & $N$ & $\begin{array}{l}\text { Media de los } \\
\text { índices por factor }\end{array}$ & $\begin{array}{l}\text { Desviación } \\
\text { estándar }\end{array}$ & $\begin{array}{l}\text { Prueba de Levene } \\
\text { Significancia }\end{array}$ & $\begin{array}{l}\text { Prueba t para } \\
\text { igualdad de medias } \\
\text { (significancia bil.) }\end{array}$ \\
\hline \multirow{2}{*}{ Creencias } & CAE & 125 & 0.4206 & 0.2161 & & \\
\hline & CME & 130 & 0.3170 & 0.2251 & 0.553 & 0.000 \\
\hline \multirow{2}{*}{ Actitudes } & AAE & 125 & 0.5353 & 0.1455 & & \\
\hline & AME & 130 & 0.2923 & 0.1628 & 0.053 & 0.000 \\
\hline \multirow{2}{*}{ Emociones } & EAE & 125 & 0.3137 & 0.1917 & & \\
\hline & EME & 130 & 0.2646 & 0.1986 & 0.560 & 0.046 \\
\hline
\end{tabular}

Fuente: Elaboración propia en SPSS. 


\section{Discusión de resultados y conclusiones}

Lo primero que puede notarse de las tablas 9, 10 y 11 , es que la prueba t-Student de comparación de medias de los índices de las emociones no juega un papel importante en cuanto a su contribución a las diferencias de los índices de AM totales, debido a que los valores $\mathrm{p}$ de la prueba t-Student, no fueron significativos en los índices totales ni en los índices de los alumnos de Administración. El valor p en los índices de los alumnos de Ingeniería es de 0.046. En consecuencia, puede decirse que, en este rubro, tanto los estudiantes alemanes como los estudiantes mexicanos son similarmente emotivos ante las matemáticas. Por consiguiente, en lo que resta, solamente nos referiremos a las contribuciones de las actitudes y las creencias a las diferencias entre los índices de AM de estudiantes mexicanos y estudiantes alemanes.

La tabla 8 muestra que, en conjunto, los estudiantes alemanes muestran una tendencia mayor a sufrir AM que los estudiantes mexicanos, debido a que los índices de AM son mayores, especialmente en el caso de Ingeniería. Las contribuciones de las creencias y de las actitudes a tal resultado se aprecian en la tabla 9, de la que observamos que las actitudes contribuyen a la AM en mayor grado que las creencias, ya que la diferencia entre las medias de los índices de las actitudes de estudiantes de ambos países es mayor a la misma diferencia para las creencias.

De la misma manera, por área de estudio (tabla 8) los estudiantes de Administración muestran mayor AM que los estudiantes de Ingeniería en ambos países. Por factor, se evidencia que las actitudes contribuyen en mayor grado que las creencias en ambos grupos de estudiantes y muy especialmente en Ingeniería, lo que corrobora el resultado global mencionado en los párrafos anteriores.

Los resultados anteriores se relacionan con las dimensiones de Hofstede y colaboradores (2010) en cuanto a que podemos clasificar algunas de ellas dentro de las categorías de creencias y actitudes. Las características de una dimensión pueden corresponder a alguno de los dos factores, creencias o actitudes. En el caso de Distancia de Poder (tabla 1), la característica 1.2 corresponde a una actitud, mientras que $1.4,1.5 \mathrm{y}$ 1.6 corresponden a creencias. Para el caso del Individualismo (tabla 2), la característica 2.1 corresponde a una actitud, mientras que las características 2.2 y 2.3 a creencias. Con respecto a Evasión de Incertidumbre (tabla 4), 4.1 es una actitud, mientras que 4.2 y 4.3 son creencias. El caso de la masculinidad no la tomaremos en cuenta, puesto que los índices numéricos de tal dimensión no presentan diferencias significativas entre ambos países (tabla 5).

Es claro que el instrumento aplicado no contempla la evaluación de las creencias y actitudes correspondientes a las dimensiones de Hofstede y colaboradores; sin embargo, podemos inferir que dentro de éstas existen dos actitudes que creemos de gran peso (la diagonal separa la actitud, primero, de estudiantes alemanes y en seguida de los estudiantes mexicanos): 1.2 Los estudiantes tratan a sus profesores como iguales/Los estudiantes muestran respeto a sus profesores, aun fuera de clase. 4.1 Los estudiantes se sienten cómodos con situaciones de aprendizaje de respuesta abierta y se interesan por buenas discusiones/Los estudiantes se sienten cómodos con aprendizajes estructurados y se interesan por la respuestas correctas. Suponemos que la influencia de estas actitudes sobre las evaluadas por el instrumento es importante por la naturaleza de las actitudes en sí mismas.

Finalmente, Hofstede y colaboradores han sido criticados por autores como McSweeney (2002) por no haber sido exhaustivos en su caracterización de los rasgos culturales. Sin embargo, creemos que algunos de los rasgos que mencionan se pueden tomar en cuenta para interpretar la AM a la luz de diferencias culturales. La discusión de resultados menciona dos actitudes, 1.2 y 4.1 de las tablas 1 y 4 , respectivamente, que son típicas de los estudiantes mexicanos encuestados. Por lo tanto, la diferencia entre valores de medias de índices de AM sugiere consistencia con algunos de los rasgos culturales de Hofstede y colaboradores. 


\section{Referencias}

Caballero Carrasco, Ana, Lorenzo J. Blanco Nieto, y Eloísa Guerrero Barona (2008), "El dominio afectivo en futuros maestros de matemáticas en la Universidad de Extremadura", en Paradigma, vol. 29, núm. 2, pp. 157-171.

Damasio, Antonio (2003), "The feeling of what happens", en R. C. Solomon (ed.), What is an emotion?, Nueva York, Oxford University Press.

Eccius-Wellmann, Cristina y Antonio Lara-Barragán Gómez (2016), "Hacia un perfil de ansiedad matemática en estudiantes de nivel superior", en Revista Iberoamericana de Educación Superior, vol. 7, núm. 18, pp. 109-129.

Engelhard, George (2001), "Math anxiety, mother's education, and the mathematics performance of adolescent boys and girls: evidence from the United States and Thailand", en The fournal of Psychology, vol. 124, núm. 3, pp. 289-298.

Fiore, Greg (1999), "Math-abused students: are we prepared to teach them?", en Mathematics Teacher, vol. 92, núm. 5, pp. 403-407.

Galbraith, Peter y Chris Haines (2000), "Mathematicscomputing attitude scales", en Monographs in Continuing Education, Londres, City University.

Gil, Nuria, Lorenzo J. Blanco y Eloísa Guerrero (2005), "El dominio afectivo en el aprendizaje de las matemáticas. Una revisión de sus descriptores básicos", en Revista Iberoamericana de Educación Matemática, núm. 2, pp. 15-32.

Gómez Chacón, Inés María (2000), Matemática emocional: los afectos en el aprendizaje matemático, Madrid, Narcea.

Goos, Merrilyn, Ray Brown y Katie Makar (eds.) (2008), "Advancing research into affective factors in mathematics learning: clarifying key factors, terminology and measurement", en Proceedings of the 31 st Annual Conference of the Mathematics Education Research Group of Australasia, MERGA Inc.

Guerrero, Eloísa, Lorenzo J. Blanco y Florencio Vicente Castro (2001), "Trastornos emocionales ante la educación matemática”, en J. N. García (coord.), Aplicaciones de intervención psicopedagógica, España, Pirámide, pp. 229-237.
Hannula, Markku S. (2002), "Attitude towards mathematics: emotions, expectations and values", en Educational Studies in Mathematics, núm. 49, pp. 25-46.

Hembree, Ray (1990), "The nature, effects, and relief of mathematics anxiety", en fournal for Research in Mathematics Education, vol. 21, núm. 1, pp. 33-46.

Hofstede, Geert, Gert Jan Hofstede y Michael Minkov (2010), Intercultural cooperation and its importance for survival, Nueva York, McGraw Hill.

Huntsinger, Carol S., Paul E. Jose, Fong-Ruey y Wei-Di Ching (1997), "Cultural differences in early mathematics learning: comparison of Euro-American, ChineseAmerican, and Taiwan-Chinese families", en International Journal of Behavioral Development, vol. 21, núm. 2, pp. 37 1-388.

Legg, Angela M. y Lawrence Locker, Jr. (2009), "Math performance and its relationship to math anxiety and metacognition", en North American fournal of Psychology, vol. 11, núm. 3, pp. 471-486.

Leppävirta, Johanna (2011), "The impact of mathematics anxiety on the performance of students of electromagnetics", en fournal of Engineering Education, vol. 100, núm. 3, pp. 424-443.

Macías-Martínez, Daniel y María del Rocío HernándezPozo (2008), "Indicadores conductuales de ansiedad escolar en bachilleres en función de sus calificaciones en un examen de matemáticas", en Universitas Psychologica, vol. 7, núm. 3, pp. 767-785.

Mato, María Dorinda y Enrique de la Torre (2009), "Actas del Primer Simposio de la SEIEM", en Investigación en Educación Matemática, Santander, Universidad de Cantabria, 285-300.

McLeod, Douglas B. (1989), "Beliefs, attitudes, and emotions: new view of affect in mathematics education", en Douglas B. McLeod y Verna M. Adams (eds.), Affect and mathematics problem solving: a new perspective, Nueva York, Springer-Verlag, pp. 245-25.

McSweeney, Brendan. (2002), "Hofstede'smodel of national cultural differences and their consequences: A triumph of faith - a failure of analysis", en Human Relations, vol. 55, núm. 1, pp. 89-118. 
Mohamed, Lawsha y Hussain Waheed (2011), "Secondary students' attitude towards mathematics in a selected school of Maldives", en International fournal of Humanities and Social Science, vol. 1, núm. 15, pp. 277-281.

Pierce, Robyn, Kaye Stacey y Anastasios Barkatsas (2007), "A scale for monitoring students' attitudes to learning mathematics with technology", en Computers \& Education, vol. 48, núm. 2, pp. 285-300.

Sloan, Tina Rye (2010), "A quantitative and qualitative study of math anxiety among preservice teachers", en The Educational Forum, núm. 74, pp. 242-256.

Soong, Hannah, Richard Lee y George John (2012), "Cultural differences in justificatory reasoning", en Educational Review, vol. 64, núm. 1, pp. 57-76.

Xie, Dong y Frederick T. Leong (2008), "A crosscultural study of anxiety among Chinese and Caucasian American university students", en fournal of Multicultural Counseling and Development, núm. 36, pp. 52-56.

\section{Cómo citar este artículo:}

Eccius-Wellmann, Cristina, Antonio G. Lara-Barragán, Bastian Martschink y Stefan Freitag (2017), “Comparación de perfiles de ansiedad matemática entre estudiantes mexicanos y estudiantes alemanes", en Revista Iberoamericana de Educación Superior (RIES), México, UNAM-IISUE/Universia, vol. VIII, núm. 23, pp. 69-83, [consulta: fecha de última consulta]. 